Article

\title{
Functional Analysis of a Putative Dothistromin Toxin MFS Transporter Gene
}

\author{
Rosie E. Bradshaw ${ }^{1, *}$, Zhilun Feng ${ }^{1}$, Arne Schwelm ${ }^{1}$, Yongzhi Yang ${ }^{2}$ and Shuguang Zhang ${ }^{1}$ \\ 1 Bio-Protection Research Centre, Institute of Molecular BioSciences, Massey University, \\ Palmerston North, New Zealand; E-Mails: fengzhilun@hotmail.com (Z.F.); \\ schwelmo@gmx.de (A.S.); s.g.zhang@massey.ac.nz (S.Z.) \\ 2 Biotechnology Research Institute, Qinghai Academy of Agriculture and Forestry, Xining, China; \\ E-Mail: zhmyyz03@yahoo.com.cn (Y.Y.) \\ * Author to whom correspondence should be addressed; E-Mail: r.e.bradshaw@massey.ac.nz; \\ Tel.: +64-6-350-5515; Fax: +64-6-350-5688.
}

Received: 13 November 2009; in revised form: 20 November 2009 / Accepted: 7 December 2009 / Published: 8 December 2009

\begin{abstract}
Dothistromin is a non-host selective toxin produced by the pine needle pathogen Dothistroma septosporum. Dothistromin is not required for pathogenicity, but may have a role in competition and niche protection. To determine how D. septosporum tolerates its own toxin, a putative dothistromin transporter, DotC, was investigated. Studies with mutants lacking a functional $\operatorname{dotC}$ gene, overproducing DotC, or with a DotC-GFP fusion gene, did not provide conclusive evidence of a role in dothistromin efflux. The mutants revealed a major effect of DotC on dothistromin biosynthesis but were resistant to exogenous dothistromin. Intracellular localization studies suggest that compartmentalization may be important for dothistromin tolerance.
\end{abstract}

Keywords: aflatoxin biosynthesis; Dothistroma septosporum; major facilitator superfamily; toxin transporter; red-band needle blight

\section{Introduction}

Dothistromin toxin is produced by fungal pine pathogens in the genus Dothistroma (class Dothideomycetes) and is responsible for the characteristic red banding seen in infected needles [1,2]. A taxonomic revision in 2004 split the pathogen Dothistroma pini into two new species: $D$. 
septosporum, that has a worldwide distribution, and D. pini that appears to be confined to a few regions in the Northern hemisphere [3]. In recent years the prevalence and severity of this disease has increased, with epidemics in Canada and Europe associated with climate change [4,5].

Dothistromin is a difuroanthraquinone toxin with a polyketide backbone. Its structure is similar to that of versicolorin B, a precursor of aflatoxin and sterigmatocystin [6]. Dothistromin is toxic not only to pine tissue, but also to a wide range of other eukaryotes and bacteria [7,8], hence is considered a non-host-selective toxin. The precise mode of action is not known but dothistromin can be reductively activated leading to the formation of the reactive oxygen species superoxide $\left(\mathrm{O}_{2}{ }^{-}\right)$and $\mathrm{H}_{2} \mathrm{O}_{2}$ [9] .

Dothistromin was once suspected to have a major role in needle blight disease. However dothistromin-deficient mutants of $D$. septosporum made by disruption of the dothistromin polyketide synthase gene ( $p k s$ ) were pathogenic to Pinus radiata, showing that dothistromin is not required for disease development. Instead the toxin may have a role in competition against other microorganisms in the needle environment, a hypothesis that is supported by competition studies in vitro [10]. But given that dothistromin is highly toxic to a wide range of fungal species, the question arises of how $D$. septosporum is able to tolerate its own toxin.

Many fungal toxin gene clusters include toxin transporter genes and some of these have been shown to have roles in virulence and/or self-protection against the toxins [11,12]. Transporters of the nonhost-selective toxin cercosporin have been implicated in virulence, including the CFP transporter in Cercospora kikuchii [13] and CTB4 in Cercospora nicotianae [14]. Disruption of genes encoding these transporters significantly lowered the amount of cercosporin produced in each case, suggesting a role in regulation of cercosporin biosynthesis. However, whilst the CFP mutants were sensitive to exogenously supplied cercosporin the CTB4 mutants showed normal levels of resistance [13-15]. Disruption of some transporter genes appears to have no effect on either production or efflux of the associated toxin, such as the AflT transporter encoded in the aflatoxin gene cluster [16]. It is likely that there is redundancy with other transporters able to complement the disruption mutations $[12,16,17]$.

Genes associated with dothistromin biosynthesis were identified on the basis of their similarity to aflatoxin genes $[18,19]$. The $\operatorname{dot} A$ gene was the first to be identified, and encodes a ketoreductase with 80\% amino acid identity to the aflatoxin Ver-1 (AflM) in Aspergillus parasiticus. Other genes found alongside $\operatorname{dot} A$ include $\operatorname{dot} C$ that encodes a putative dothistromin transporter in the major facilitator superfamily (MFS), with 14 transmembrane domains. DotC is similar in sequence to MFS proteins in other species in the Dothideomycetes. It has 53\% amino acid identity with both the Fnx1 multidrug resistance protein of Phytophthora tritici-repens (XP_001938334) and a hypothetical protein (SNOG_04383) in Phaeosphaeria nodorum (XP_001794802). In contrast there is less than 32\% amino acid identity to Aspergillus parasiticus AflT (AAS66020.1), Cercospora kikuchii CFP (AAC78076) and Cercospora nicotianae CTB4 (ABK64181.1).

The aim of this investigation was to analyse the function of DotC to determine if it has a role in tolerance, efflux and/or biosynthesis of dothistromin. Mutants with a targeted disruption of $\operatorname{dot} C$, or a complemented strain with multiple copies of $\operatorname{dot} C$, were studied along with strains expressing DotC-GFP or DotA-GFP fusion proteins in order to determine the location and role of DotC in $D$. septosporum. 


\section{Results and Discussion}

\subsection{Production and characterization of $\mathrm{D}$. septosporum dotC mutants and complemented strains}

D. septosporum strains transformed with a $\operatorname{dot} C$ replacement construct (pR260) were selected by hygromycin resistance. Two out of 26 hygromcyin resistant colonies (named FJT15 and FJT16) showed correct targeted replacement of the $\operatorname{dot} C$ gene when tested by PCR (results not shown) and Southern blotting (Figure 1). Other transformants had ectopic integration of the construct or vector integration only in the 3' region of $\operatorname{dot} C$. The Southern blot also confirmed that there is only one copy of $\operatorname{dot} C$ in the $D$. septosporum genome. The $\operatorname{dot} C$ mutant FJT15 was subsequently transformed with a complementation construct (pR282) to restore $\operatorname{dot} C$ function and integration of the construct in the complemented strain FJT93 was confirmed by PCR (results not shown). The copy number of $\operatorname{dot} C$ in the complemented strain was estimated by real-time PCR to be 10 copies, making FJT93 a DotC-overproducing strain.

No significant difference was seen between sporulation of the $\operatorname{dotC}$ mutants FJT15 $\left(1.48 \pm 0.32 \times 10^{6}\right.$ spores $\left./ \mathrm{mL} ; \mathrm{P}=0.725\right)$ and FJT16 $\left(1.17 \pm 0.29 \times 10^{6}\right.$ spores $\left./ \mathrm{mL} ; \mathrm{P}=0.706\right)$ compared to the wild type NZE10 $\left(1.32 \pm 0.29 \times 10^{6}\right.$ spores $\left./ \mathrm{mL}\right)$. Likewise the radial growth rate of the $\operatorname{dot} C$ mutants was similar to that of the wild type with FJT15 $(0.60 \pm 0.03 \mathrm{~mm} /$ day; $\mathrm{P}=0.092)$ and FJT16 $(0.58 \pm 0.02 \mathrm{~mm} /$ day; $\mathrm{P}=0.182)$ not significantly different from the wild type $(0.54 \pm 0.02 \mathrm{~mm} /$ day $)$. The $\operatorname{dotC}$-complemented strain FJT93 grew slightly slower than the wild-type but in these experiments the difference was not significant $(0.50 \pm 0.02 \mathrm{~mm} / \mathrm{day} ; \mathrm{P}=0.119)$.

Figure 1. KpnI-digested genomic DNA of D. septosporum wild-type (lanes 1 \& 2) or transformed with pR260 (lanes 3-8) and hybridized with a $\operatorname{dot} C$ coding region probe. KpnI sites flank the $\operatorname{dot} C$ gene, hence replacement of a $1.4 \mathrm{~kb}$ central portion of $\operatorname{dot} C$ with a $2.4 \mathrm{~kb}$ hygromycin resistance gene cassette increased the $\operatorname{dot} C$-containing $\mathrm{KpnI}$ fragment size from $3.5 \mathrm{~kb}$ (wild-type) to $4.5 \mathrm{~kb}$ ( $\operatorname{dot} C$ replacement). The $\operatorname{dot} C$ replacement mutants FJT16 and FJT15 are shown in lanes 3 and 8 respectively. Lane 6 contains another $\operatorname{dot} C$ mutant that had an additional ectopic integration of the pR260 plasmid.

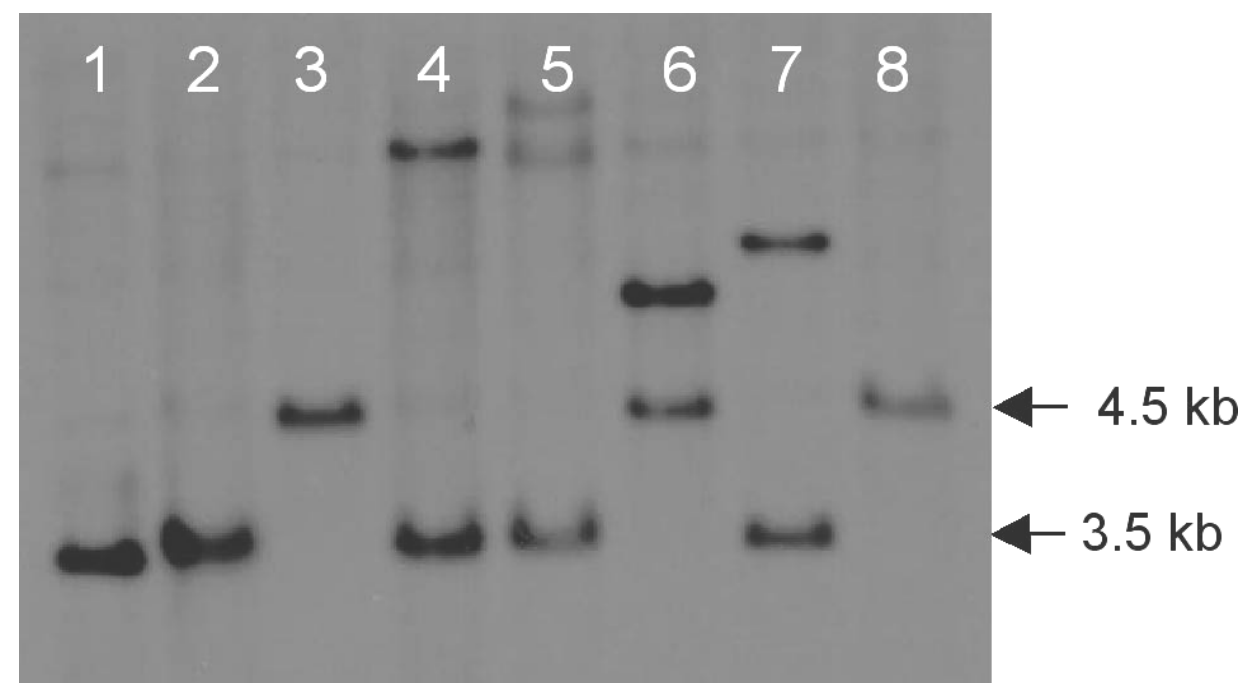




\subsection{Resistance of mutants to exogenous dothistromin}

To determine if DotC confers a protective effect against exogenously supplied toxin, the ability of $\operatorname{dot} C$ mutants to grow in the presence of dothistromin was investigated (Table 1). Although there was some variability in growth rates, as often seen with $D$. septosporum, none of the strains appeared to be strongly inhibited by even the highest concentration of dothistromin. Colonies on $100 \mu \mathrm{M}$ dothistromin grew to $>96 \%$ of the diameter of colonies grown without dothistromin. This concentration of dothistromin is in excess of levels usually secreted by the strains $(5-11 \mu \mathrm{M}$ for NZE10 and 20-40 $\mu \mathrm{M}$ secreted by FJT93). Hence it appears that DotC is not required for resistance to dothistromin in D. septosporum. Similarly in C. nicotianae CTB4 mutants showed normal levels of resistance to exogenously supplied cercosporin, although disruption of other putative toxin pump genes in Cercospora spp. (CFP in C. kikuchii and ATRI and CnCFP in C. nicotianae) had some effect on cercosporin resistance [13-17].

In these experiments using a total diameter calculation the complemented (DotC-overproducing) FJT93 strain did show significantly slower growth than the wild type $(\mathrm{P}<0.01)$ in all concentrations of dothistromin, in contrast to the earlier study.

Table 1. Growth of D. septosporum in the presence of exogenous dothistromin (doth). Mean radial growth $(\mathrm{mm}) \pm$ standard error shown for two independent experiments each with 10 replicates. *Significant differences between growth of each strain with dothistromin compared to without dothistromin $(\mathrm{P}<0.05)$ are indicated by asterisks.

\begin{tabular}{lllll}
\hline \multicolumn{1}{c}{ Strain } & $\mathbf{0} \boldsymbol{\mu M}$ doth & $\mathbf{2 0} \boldsymbol{\mu M}$ doth & $\mathbf{4 0} \boldsymbol{\mu M}$ doth & $\mathbf{1 0 0} \boldsymbol{\mu M}$ doth \\
\hline NZE10 (wildtype) & $14.7 \pm 0.19$ & $14.6 \pm 0.27$ & $14.0 \pm 0.11^{*}$ & $14.2 \pm 0.12^{*}$ \\
FJT15 $(\Delta \operatorname{dot} C)$ & $15.5 \pm 0.43$ & $16.1 \pm 0.30$ & $16.1 \pm 0.36$ & $16.7 \pm 0.33^{*}$ \\
FJT16 $(\Delta \operatorname{dot} C)$ & $17.3 \pm 0.29$ & $16.6 \pm 0.23$ & $16.4 \pm 0.25^{*}$ & $16.9 \pm 0.20$ \\
FJT93 $(\Delta \operatorname{dot} C+\operatorname{dot} C)$ & $13.4 \pm 0.21$ & $12.6 \pm 0.14^{*}$ & $13.2 \pm 0.18$ & $13.3 \pm 0.21$ \\
\hline
\end{tabular}

\subsection{Dothistromin production by dotC disruption mutants}

Amounts of dothistromin secreted into broth, and remaining in the mycelium, were determined by ELISA following a standardised extraction procedure (Table 2). Strikingly, the $\operatorname{dot} C$ disruption mutants (FJT15 and FJT16) secreted only 4-7\% of the amount of dothistromin secreted by the wild type NZE10 strain. The amounts of dothistromin in the mycelia were also lower in these mutants than the wild-type, but these differences were not significant, possibly due to large variation between replicates.

It is possible that the lower levels of secreted dothistromin in the $\operatorname{dot} C$ mutants were a direct consequence of overall lower levels of dothistromin production. The amounts of dothistromin produced by the mutants (mycelium and broth combined) were only $8.5 \%$ and $4.4 \%$ of wild type levels for the FJT15 and FJT16 mutants respectively. However the right-hand column in Table 2 suggests a small, but not significant, difference in the percentage of dothistromin secreted: in the wild type over $90 \%$ of all dothistromin was secreted whilst in the $\operatorname{dot} C$ knockout mutants only $80-83 \%$ was secreted. Thus there is only weak evidence for a role in dothistromin transport for DotC. 
Table 2. Dothistromin as measured by ELISA from broth (secreted dothistromin) and mycelium (not secreted) extracted from the same flasks using the same extraction method $(\mathrm{n}=3)$. For mycelium the amounts shown are dothistromin per mg dry weight. For broth the amounts of dothistromin have been calculated to show how much dothistromin was secreted into the broth by each mg of mycelium in the flask. *Significant differences from NZE10 values $(\mathrm{P}<0.05)$ are indicated by an asterisk.

\begin{tabular}{|c|c|c|c|c|c|}
\hline \multirow[b]{2}{*}{ Strain } & \multicolumn{2}{|c|}{ Dothistromin in broth } & \multicolumn{2}{|c|}{ Dothistromin from mycelia } & \multirow[b]{2}{*}{$\begin{array}{c}\text { \% Doth secreted } \\
\text { broth/(myc + } \\
\text { broth) }\end{array}$} \\
\hline & $\begin{array}{c}\text { Dothistromin } \\
\text { Mean } \pm \mathrm{SE} \\
(\mathrm{ng} / \mathrm{mg} \mathrm{DW})\end{array}$ & $\begin{array}{c}\% \\
\text { of WT } \\
(\mathrm{NZE} 10) \\
\end{array}$ & $\begin{array}{l}\text { Dothistromin } \\
\text { Mean } \pm \mathrm{SE} \\
(\mathrm{ng} / \mathrm{mg} \mathrm{DW})\end{array}$ & $\begin{array}{c}\% \\
\text { of WT } \\
\text { (NZE10) }\end{array}$ & \\
\hline NZE10 (wildtype) & $319.8 \pm 30.3$ & 100 & $30.5 \pm 15.3$ & 100 & $91.3 \%$ \\
\hline NZE7 (wildtype) & $249.1 \pm 32.8$ & 77.9 & $26.8 \pm 16.6$ & 88.1 & $90.3 \%$ \\
\hline FJT15 $(\Delta d o t C)$ & $23.8 \pm 5.0^{*}$ & 7.4 & $6.1 \pm 2.2$ & 20.1 & $79.5 \%$ \\
\hline FJT16 $(\Delta \operatorname{dot} C)$ & $12.9 \pm 2.7 *$ & 4.0 & $2.6 \pm 0.6$ & 8.4 & $83.4 \%$ \\
\hline FJT93 $(\Delta \operatorname{dot} C+\operatorname{dot} C)$ & $1716.6 \pm 507.9$ & 536.8 & $1105.3 \pm 348.2^{*}$ & 3629.9 & $60.8 \% *$ \\
\hline
\end{tabular}

The lower levels of dothistromin in the $\operatorname{dot} C$ mutants strongly suggest that DotC is required for wild-type levels of dothistromin biosynthesis. DotC is a close match to MFS proteins, with no evidence for a DNA binding domain. A dramatic reduction in toxin production was also seen in CFP mutants of Cercospora kikuchii, with less than 5\% of wild type levels of cercosporin produced [13], and also to CTB4 and ATR1 mutants of C. nicotianae with less than $35 \%$ and $25 \%$ of wild type cercosporin respectively $[14,17]$.

The observation that some dothistromin was still secreted in the $\operatorname{dot} C$ mutants suggests that other transporters are involved. Multiple transporters with overlapping roles have been reported in several fungi. For example in $C$. nicotianae the $\mathrm{ABC}$ transporter ATR1 was shown to have a major role in cercosporin production and, along with another MFS transporter CnCFP, contributed to auto-resistance to cercosporin [17].

The $\operatorname{dot} C$ complemented mutant (FJT93) produced and secreted very high levels of dothistromin. The secreted levels were five-fold higher than those seen with the wild-type, but the variability between replicates was high. The amount of dothistromin secreted from FJT93 was significantly higher than the combined results from wild type strains NZE7 and NZE10 ( $\mathrm{P}=0.0037)$ but not compared to NZE10 alone $(\mathrm{P}=0.0516)$. The levels of non-secreted dothistromin remaining in the mycelium were extremely high in FJT93: more than 30-fold higher than the wild type. It appears there was a dramatic increase in dothistromin biosynthesis in FJT93 and that the cells were unable to secrete the dothistromin as quickly as it was being made: almost $40 \%$ of the total amount of dothistromin remained in the mycelium. Since FJT93 only showed a slightly slower growth rate compared to the wild type (significant in only one of two trials) the high intracellular levels of dothistromin did not appear to be toxic. These high levels of toxin further suggest that dothistromin accumulation in the cell is not a primary feedback regulator of dothistromin biosynthesis. 


\subsection{Dothistromin gene expression in dotC disruption and complemented mutants}

Real-time PCR was used to quantify $\operatorname{dot} C$ expression in the $\operatorname{dot} C$ mutants and complemented strain. As expected, the $\operatorname{dot} C$ disruption mutants FJT15 \& 16 showed no $\operatorname{dot} C$ expression above background, as they lacked this single-copy gene. Expression of the dotA dothistromin biosynthetic gene was significantly reduced in the $\operatorname{dot} C$ mutants, to around $18-26 \%$ of levels in wild type strains (Figure 2 and Supplementary Table S3). It therefore seems likely that the lower levels of dothistromin made by the mutants are at least partly due to lower levels of biosynthetic enzymes. Both FJT15 and FJT16 also showed different tubA (constitutive control) expression levels compared to the wild type, but one had a higher and one a lower level of $t u b A$ so no consistent effects were noted. The $\operatorname{dot} C$ mutants showed lower levels of expression of the dothistromin biosynthetic genes $p k s A$ and $v b s A$, but the NZE7 wild type strain also had reduced levels of these transcripts compared to the NZE10 wild type. NZE7 and NZE10, like all New Zealand isolates of D. septosporum, are considered clonal but strain degeneration has sometimes been noted over time in axenic culture. The NZE7 strain was isolated from infected pine needles three years before NZE10 and the differences in gene expression may reflect this. Interstrain differences were also noted between the independent dotC mutants FJT15 and FJT16, making the $p k s A$ and $v b s A$ results less reliable than those for $\operatorname{dot} A$.

Figure 2. Expression of $\operatorname{dot} C$, dothistromin genes $\operatorname{dot} A, p k s A$ and $v b s A$, and beta tubulin (tubA, constitutive control) in wild type, $\operatorname{dot} C$ knockout $(\triangle \operatorname{dot} C)$ and complemented $(\Delta \operatorname{dot} C+\operatorname{dot} C)$ strains. $18 \mathrm{~S}$ ribosomal RNA was used as a reference gene for standardisation. Values are normalised expression ratios relative to the NZE10 wild type, shown as mean \pm standard error $(\mathrm{n}=6)$. *Significant differences from NZE10 $(\mathrm{P}<0.05)$ are shown by an asterisk.
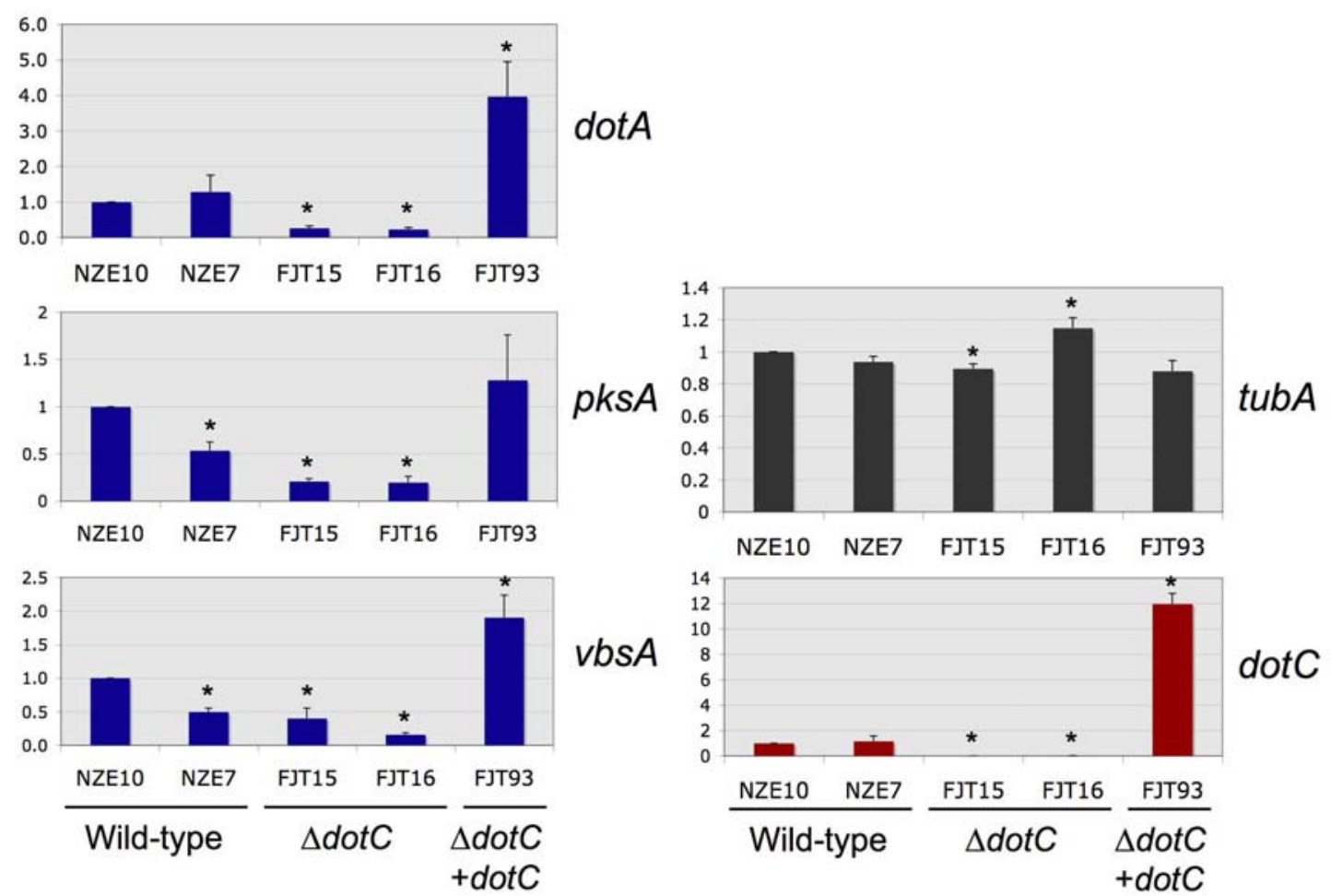
In C. nicotianae, although disruption of the CTB4 MFS gene led to significantly lower levels of cercosporin biosynthesis compared to the wild type, expression of the four cercosporin biosynthetic genes tested was not noticeably reduced in northern blots [14]. Likewise disruption of the aflT gene in A. parasiticus had no effect on the expression of aflatoxin biosynthetic genes, as well as no effect on aflatoxin production [16].

In contrast to the $\operatorname{dot} C$ mutants, the complemented FJT93 strain that contained multiple copies of $\operatorname{dot} C$ produced 12-fold more $\operatorname{dot} C$ transcript than the wild type, suggesting over-expression of DotC protein. It is possible that the $\operatorname{dot} C$ complementation construct may have integrated into a site that affects expression of dothistromin genes. However the increased gene expression is consistent with a role for DotC in regulating dothistromin biosynthesis and with the decline in dothistromin production seen in the $\operatorname{dot} C$ disruption mutants. Further to this, high intracellular concentrations of dothistromin in a strain that appears to make an excess of DotC adds support to the hypothesis that DotC is not the major efflux pump for dothistromin and suggests that there are other factors besides DotC limiting secretion.

The FJT93 complemented strain also over-expressed two of the dothistromin biosynthetic genes ( $\operatorname{dot} A$ and $v b s A$ ), although levels were only 2-4 fold higher than the wild type. The fact that $p k s A$ gene expression was not significantly increased in the DotC and dothistromin over-expressing mutant is interesting because $p k s A$ encodes a polyketide synthase required for an early step of dothistromin biosynthesis. Since the $p k s A$ gene is essential for dothistromin production [20] this result suggests that PksA is not a major rate-limiting enzyme in dothistromin biosynthesis.

\subsection{Cellular localization of DotC-GFP and DotA-GFP fusion proteins}

Confocal microscopy showed that the DotC-GFP fusion protein was located around the periphery of the cells (Figure 3), as would be expected for an MFS transporter. This is consistent with a role in secretion of dothistromin. However the images also revealed DotC-GFP localization in some intracellular regions. Cross-sections through these suggested that DotC-GFP was not confined to the perimeter but seen throughout. These GFP-containing regions varied in intensity and size but were reminiscent of vacuoles containing the aflatoxin pathway enzymes Ver-1 or Nor-1 fused to GFP $[21,22]$. Under aflatoxin-inducing conditions in A. parasiticus a large number of vesicles accumulated that were heterogeneous in both density and size, and a functional link of vesicles (defined as vacuolar-type structures with diameters $<2.5 \mu \mathrm{m}$ ) and vacuoles with aflatoxin biosynthesis was proposed [23].

Cellular localisation of the DotA dothistromin biosynthetic enzyme was also studied in a $D$. septosporum strain expressing a DotA-GFP fusion. The DotA-GFP fusion protein was predominantly cytoplasmic or located in small (Figure 3c) or larger (Figure 3d) vesicles, and showed similarities to the localization of GFP fusion genes of $A$. parasiticus aflatoxin genes, including the DotA homolog, Ver-1 [21,22]. The Ver-1 fusion protein was predominantly cytoplasmic at early stages of growth under aflatoxin-inducing conditions and found in the lumen of up to $80 \%$ of vesicle-like structures and vacuoles at later stages [21]. Studies with a Nor-1 fusion showed a similar trend [22]. In the present study $D$. septosporum cultures were observed after 4-5 days of growth (early exponential phase), so it is possible that DotA-GFP was in the early stages of moving to vacuoles. 
Figure 3. GFP fluorescence of D. septosporum transformed with $\operatorname{dot} C-g f p(\mathbf{a}, \mathbf{b})$ or $\operatorname{dotA}$ $g f p$ (c, d). Confocal Laser Scanning Microscopy (CLSM) images on the left (a and c) are combined $0.2 \mu \mathrm{m} \mathrm{Z}$-stacks. Images on the right (b and d) are single-layer snapshots, with vertical cross-section profiles illustrated on the right. White arrows indicate intracellular vesicles/vacuoles showing GFP-fluorescence. Vesicles in (c) are very small. Scale bar $=20 \mu \mathrm{m}$.
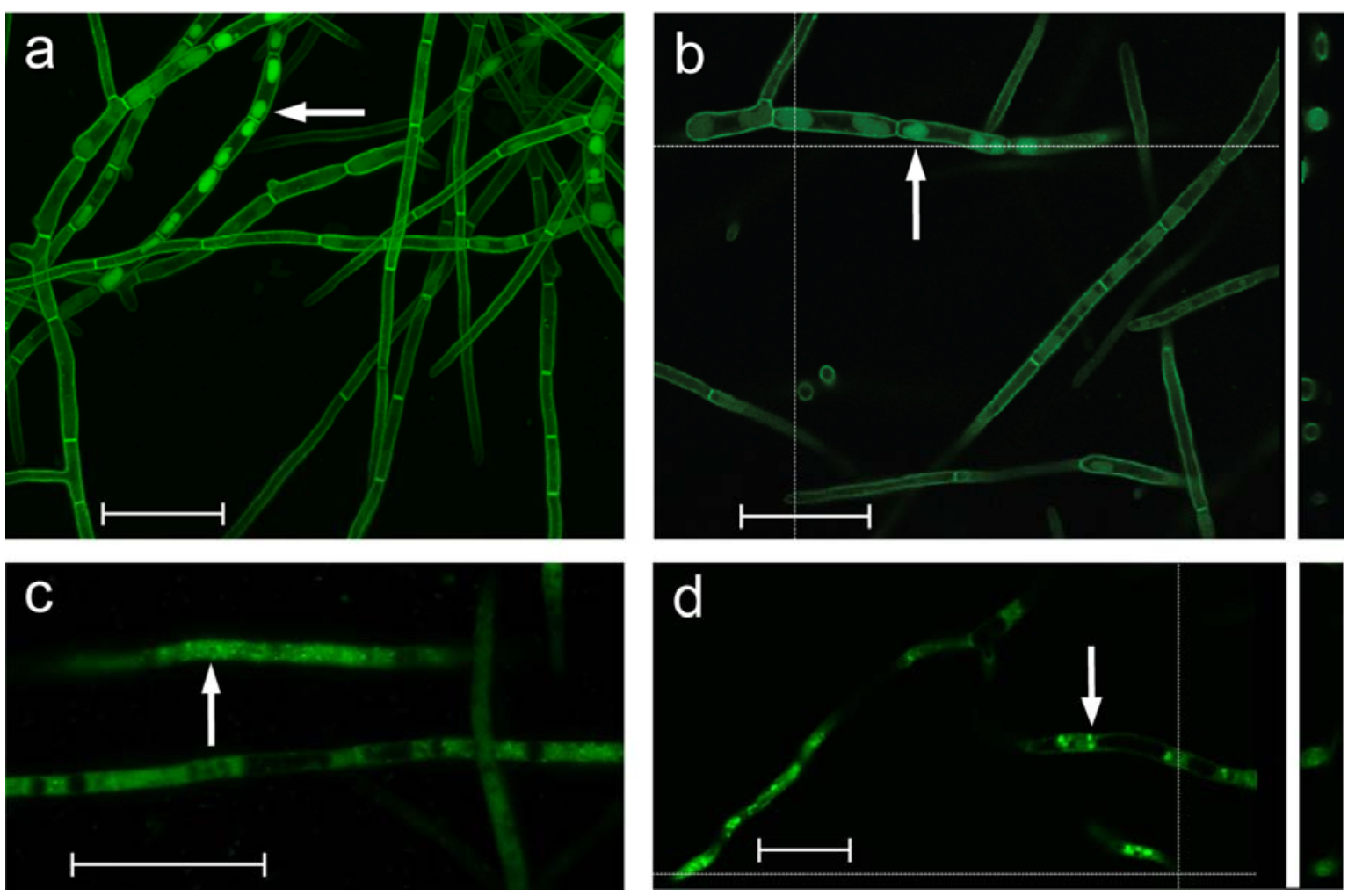

Although most of the DotC-GFP protein was localized in the cell membrane and therefore could be involved in efflux from the cell, its localisation in some intracellular organelles might suggest an additional role. We propose the hypothesis that mid to late stages of dothistromin biosynthesis occur in vacuoles as proposed for aflatoxin biosynthesis [21] and that this enables $D$. septosporum to tolerate high intracellular toxin levels as seen in the over-expressing strain FJT93. We further propose the hypothesis that one function of DotC may be to transport early-stage dothistromin biosynthetic intermediates from the cytoplasm into vacuoles, thereby affecting the rate of dothistromin production. This could explain the lower levels of dothistromin produced by $\operatorname{dot} C$ mutants and higher levels in the DotC over-producing strain. The CnCFP transporter in C. nicotianae was also speculated to have a role in transport between organelles and/or transport of early pathway components within the cell [17], although in this case no cellular localization studies were reported.

There are some anomalies in our data that make interpretation difficult. Cross-sections of vacuoles showed DotC-GFP throughout some of the vacuoles, rather than just at the periphery as would be expected if DotC has a role in intracellular transport. Furthermore the dotA-egfp gene did not function as expected: it was transformed into a $\operatorname{dot} A^{-}$dothistromin-deficient mutant strain (FJT2) and did not re-establish dothistromin production by complementation (results not shown). Therefore the observations of DotA-GFP expression might be artifacts, although the similarity to the aflatoxin 
biosynthesis proteins in A. parasiticus is intriguing. Further detailed studies of intracellular localization are required to determine the locations of dothistromin and the dothistromin biosynthetic enzymes, as well as the role of DotC in dothistromin biosynthesis.

According to Amnuaykanjanasin [17], fungal transporters can be classified into three groups based on whether they have a major role in toxin efflux (group 3), self-protection (group 2) or both (group 1). In $C$. nicotianae the cercosporin transporters ATR1 and CnCFP were classified as group 1 whilst CTB4, the transporter located in the cercosporin gene cluster, was group 3. On the basis of our results the D. septosporum DotC that is encoded within the dothistromin gene cluster appears to be closest to a group 3 transporter as it may be involved in dothistromin efflux but has little or no effect on protection against exogenous dothistromin. If dothistromin is compartmentalized within the cell as hypothesized above, this may contribute to self-protection, providing one possible explanation for how the DotC-overproducing strain tolerated extremely high intracellular levels of dothistromin without any severe effects.

An additional role that has now been noted with several fungal transporters is that of regulating toxin biosynthesis [17]. Our results clearly showed a dramatic decrease in dothistromin biosynthesis in DotC-deficient mutants and increase in a DotC-overexpressing strain. The most obvious way in which transporters might affect biosynthesis would be by a feedback mechanism due to intracellular toxin levels which would increase in mutants with deficient efflux systems. However our results suggest that a more complex type of regulation may occur. In the DotC-overproducing strain extremely high levels of dothistromin were accompanied by increased expression of dothistromin biosynthetic genes, rather than decreased expression as would be predicted from a simple dothistromin feedback regulation model. Compartmentalisation of toxin biosynthesis within the cell, as shown for A. parasiticus [21-23], and supported by our studies with D. septosporum, may prove to be an important factor in regulation of toxin biosynthesis.

\section{Materials and Methods}

\subsection{Strains and culture conditions}

The wild-type D. septosporum strains NZE7 and NZE10 (clonal forest isolates from New Zealand), and genetically modified strains derived from these, were routinely grown as previously described [10]. For radial growth measurements, 5-mm diameter agar plugs from 7-day old colonies were inoculated onto plates of Dothistroma Medium [24] and incubated at $22{ }^{\circ} \mathrm{C}$. In three independent experiments, each with 3-7 replicates, radial growth was measured at intervals and a growth rate ( $\mathrm{mm} /$ day) calculated over the $2-4$ week period since inoculation in which growth rate was constant. To assess sporulation, colonies were grown as above but on Dothistroma sporulation medium [24]. After 3 weeks' incubation, two 5-mm diameter agar plugs of mycelium were taken from opposite edges of each colony (from three independent experiments, each with 3-7 replicates), vortexed in $200 \mu \mathrm{L} 1 \%$ Tween 20, and spore concentrations determined using a cytometer. Results are presented as overall means \pm SEM unless otherwise indicated. A two-tailed T-test was used to determine significant differences based on the null hypothesis of no difference between each mutant and the wild type. 


\subsection{Disruption and complementation of $\operatorname{dotC}$}

To disrupt the $\operatorname{dot} C$ gene, a replacement vector pR260 was prepared using a Multisite Gateway ${ }^{\mathrm{TM}}$ system (Invitrogen) using methods previously described for D. septosporum pksA and vbsA genes $[19,20]$. Three entry clones were prepared by PCR amplification: (a) a 5' region of $\operatorname{dot} C$, including nucleotides 19180 to 20313, numbered according to GenBank entry AF448056, (b) a hygromycin resistance $(h p h)$ selectable marker gene cassette and (c) a 3' region of $\operatorname{dot} C$, including nucleotides 16783 to 17782 . All primers used in this work are listed in Supplementary Table S1. The flanking regions were designed so that approximately $1.4 \mathrm{~kb}$ of the $1.9 \mathrm{~kb} \operatorname{dot} C$ ORF would be replaced by the $h p h$ gene following recombination of the entry clones. The arrangement of the three fragments in the gene replacement plasmid, pR260, was confirmed by PCR and sequencing. Protoplasts of wild type $D$. septosporum NZE10 were transformed with plasmid pR260 using methods described previously [25].

Hygromycin-resistant transformants were single-spore purified and characterized using methods described previously [20]. DNA extracted from transformants was PCR-amplified to determine if $\operatorname{dot} C$ gene replacement had occurred. PCR primers were designed to amplify between the $h p h$ region of the pR260 construct and either 5' or 3' genomic regions flanking the $\operatorname{dot} C$ integration site (see Supplementary Figure S1 for details). Southern hybridisation of XbaI and BamHI-digested genomic DNA was used to confirm gene replacement using a digoxigenin (DIG)-labeled probe designed to cover the $\operatorname{dot} C \mathrm{ORF}$.

Complementation of one of the $\operatorname{dot} C$ disruption mutants (FJT15) with the complete $\operatorname{dot} C$ gene was achieved using a $4.75 \mathrm{~kb}$ HindIII-NotI genomic DNA fragment that contained the complete $\operatorname{dot} C$ coding region and approximately $2 \mathrm{~kb}$ of upstream (intergenic) sequence. The fragment was ligated into the pBC-phleo vector that contains a phleomycin selectable marker [26] to form the plasmid construct pR282, then transformed into FJT15 using selection with $7 \mu \mathrm{g} / \mathrm{mL}$ phleomycin (Apollo Scientific Ltd., Stockport, UK). Transformants were single-spore purified and characterized by PCR. To determine the copy number of the $\operatorname{dot} C$ gene in the complemented strain FJT93, quantitative realtime PCR was carried out to compare $\operatorname{dot} C$ (target) and tubA ( $\beta$-tubulin gene; normaliser) targets compared to the NZE10 wild type, using primers listed in Supplementary Table S2. Relative quantitative RT-PCR was performed using a LightCycler ${ }^{\circledR} 480$ SYBR Green 1 DNA Master kit (Roche). One $\mu \mathrm{L}$ of gDNA and $1 \mu \mathrm{L}$ primer mix (50 $\mu \mathrm{M}$ each) were added to $8 \mu \mathrm{L}$ Roche PCR mix and subjected to 45 cycles of PCR $\left(10 \mathrm{~s}\right.$ at $95{ }^{\circ} \mathrm{C}, 10 \mathrm{~s}$ at $57{ }^{\circ} \mathrm{C}, 20 \mathrm{~s}$ at $\left.72{ }^{\circ} \mathrm{C}\right)$ with an acquisition temperature of $72{ }^{\circ} \mathrm{C}$. Copy number was determined using the $2^{\Delta \Delta \mathrm{CT}}$ method and LightCycler ${ }^{\circledR} 480$ software with amplification efficiencies computed using standard curves.

\subsection{Quantification of dothistromin and dothistromin resistance assay}

Intracellular and secreted levels of dothistromin were determined for wild type, dotC mutants and complemented strains. Approximately $1 \times 10^{5}$ conidia $/ \mathrm{mL}$, harvested from DSM plates, were inoculated into $25 \mathrm{~mL}$ of dothistroma broth (DB) $(2.5 \%$ (w/v) Oxoid malt extract, $2 \%(\mathrm{w} / \mathrm{v})$ Oxoid nutrient broth) in $125 \mathrm{~mL}$ conical flasks and incubated at $22{ }^{\circ} \mathrm{C}$ for 10 days with shaking at $150 \mathrm{rpm}$. Mycelium was harvested by vacuum filtration. For each strain, three biological replicates were assessed, and a known proportion of the mycelium from each flask was freeze-dried for dry weight determination. To extract intracellular dothistromin, 35-360 mg fresh weight of mycelium was disrupted using a Thermo Savant 
FastPrep FP120 cell disrupter (Thermo Electron Corporation, Milford, MA) with $300 \mu \mathrm{L} \mathrm{H}_{2} \mathrm{O}$ and $\sim 20 \mu \mathrm{L}$ beads for ten bursts of $20 \mathrm{~s}$ at speed level 5 . An equal volume of chloroform was added, mixed for 4 hours at room temperature, the chloroform phase was collected then the aqueous phase re-extracted with chloroform four times more. The chloroform was evaporated and the residue re-dissolved in $200 \mu \mathrm{L}$ ethyl acetate, then the solvent evaporated again and the residue that contained dothistromin finally dissolved in $20-50 \mu \mathrm{L}$ DMSO. To maintain consistency, dothistromin was extracted from $5 \mathrm{~mL}$ of culture filtrate using the same method as described for the mycelium, except without the cell disruption step. Dothistromin concentrations per $\mathrm{mL}$ broth were determined using competitive ELISA [27] as previously described [24]. A direct comparison of intracellular and secreted dothistromin was made by calculating the amount of dothistromin secreted from $1 \mathrm{mg}$ dry weight mycelium, based on the total amount of mycelium in a known volume in each flask. Dothistromin resistance was assessed by radial growth on Dothistroma Medium [24] containing either 0, 20, 40 or $100 \mu \mathrm{M}$ dothistromin in DMSO, plus additional DMSO to make a final concentration of $0.08 \%$ DMSO in all plates. Five point-inoculations were made on each of two replicate plates using a well-sporulating inoculum, and toothpicks instead of the usual agar plug method, a modification suggested by Amnuaykanjanasin et al [17]. Radial growth measurements were made after 28 days growth at $22{ }^{\circ} \mathrm{C}$. The experiment was done twice.

\subsection{Real-time PCR analysis of dothistromin gene expression}

RNA was extracted from samples taken from the same cultures used for dothistromin assays, using Trizol Reagent (Invitrogen) and cDNA synthesized by random hexamer primed reverse transcription using Superscript ${ }^{\mathrm{TM}}$ III reverse transcriptase (Invitrogen). Relative quantitative RT-PCR was performed using a LightCycler ${ }^{\circledR} 480$ SYBR Green 1 DNA Master kit (Roche) as previously described [28]; (primers in Supplementary Table S2) but with amplification conditions modified to suit the LightCycler $^{\circledR} 480$. Two $\mu \mathrm{L}$ of 10 -fold diluted cDNA were added to $8 \mu \mathrm{L}$ PCR mix and subjected to 45 cycles of PCR $\left(10 \mathrm{~s}\right.$ at $95{ }^{\circ} \mathrm{C}$, then $30 \mathrm{~s}$ at each of $60{ }^{\circ} \mathrm{C}$ and $\left.72{ }^{\circ} \mathrm{C}\right)$ with an acquisition temperature of $72{ }^{\circ} \mathrm{C}$. Three technical replicates of each of two biological replicates were used for calibratornormalized relative quantification analysis. The calibrator was $18 \mathrm{~S}$ ribosomal RNA and the values were normalized by comparing to expression levels in the NZE10 wild type, with the constitutivelyexpressed $t u b A$ (beta-tubulin) gene as an internal control.

\subsection{Construction and analysis of DotC-EGFP and DotA-EGFP fusion strains}

The $\operatorname{dotC}$-egfp and dotA-egfp fusion genes were obtained using three-step combinatorial PCR, using Platinum ${ }^{\circledR}$ Taq DNA Polymerase High Fidelity (Invitrogen). In the first step for the construction of the $\operatorname{dot} C$-egfp fusion gene, a $3 \mathrm{~kb}$ DNA fragment (including the $\operatorname{dotC}$ ORF and a $1.1 \mathrm{~kb}$ upstream region) was PCR amplified from D. septosporum genomic DNA using primers dotCzf1 and dotCzf2 (Supplementary table $\mathrm{S} 1$ ). In the second step, an $0.8 \mathrm{~kb}$ fragment containing the egfp (green fluorescent protein) gene was amplified from plasmid pPN82 [29] using primers dotCzf3 and pPN812978rev. Primers dotCzf2 and primer dotCzf3 used in steps 1 and 2 were complementary and designed to match both the end of $\operatorname{dot} C$ and beginning of egfp coding regions, but with the stop codon of $\operatorname{dot} C$ eliminated to allow translation read-through into egfp. The third PCR used products from the first two 
PCR reactions as template with dotCzfl and pPN81-2978rev primers to amplify the complete fusion construct. The accuracy of the construct was verified by sequencing and it was cloned in a pBlueScript vector along with a hygromycin resistance ( $h p h$ ) selectable marker cassette (as above) to form plasmid pR265. Protoplasts of D. septosporum were transformed with plasmid pR265 as above. Purified transformants were screened for the presence of the dotC-egfp construct using PCR primers targeted to $\operatorname{dot} C$ (DSCE1) and egfp (egfp-rev) and by Southern hybridisation of XhoI-digested genomic DNA with a 420 bp DIG-labelled egfp gene fragment using methods described previously [20].

In the first step of the construction of the dotA-egfp fusion gene a $2 \mathrm{~kb}$ DNA fragment (including the dotA ORF and a $1.1 \mathrm{~kb}$ upstream region) was PCR amplified from D. septosporum using primers PdotA-fus and egfp-dota-c. In the second step a $736 \mathrm{bp}$ product was PCR amplified from plasmid pPN81 (a modified version of pPN82 [29] without the $\operatorname{trpC}$ terminator) using primers dota-egfp-c and TtrpC-fus. The primers dotA-egfp-c and egfp-dotA-c were complementary and designed to match both the end of the $\operatorname{dot} A$ and the beginning of the egfp coding regions, but replacing the $\operatorname{dot} A$ stop codon by the start codon of the egfp gene. The third step used the first two PCR products as a template and pdotA-fus and TtrpC-fus primers to PCR amplify the complete dotA-egfp fusion gene. The PCR product was cloned into pGEMTeasy (Promega) according to the manufacturer's instructions. The accuracy was verified by sequencing. A HindIII fragment from pPN82 containing the $h p h$ gene was inserted into a single restriction site resulting in vector $\mathrm{pR} 261$. The insertion of the $h p h$ gene reduced the upstream region of the $\operatorname{dot} A$ gene from 1130 to $1079 \mathrm{bp}$. Protoplast-based transformation was used to co-transform a dothistromin deficient strain $\operatorname{dot}^{-}$strain (FJT2) with pR261 and pBC-Phleo containing a selectable marker conferring phleomycin resistance [26], as described previously [10].

A strain of D. septosporum (FJT22) [28] containing a constitutively-expressed egfp gene was used as a control. For microscopic analysis, spores of D. septosporum transformants were inoculated into $15 \mathrm{~mL}$ DB broth in Petri dishes and incubated for $4-5$ days at $22{ }^{\circ} \mathrm{C}$. Fluorescence of hyphal samples was firstly assessed using an Olympus BX51 microscope with excitation and emission filters set at 460-490 nm and 510-550 nm respectively. Samples were then analysed with a Leica TCS SP5 confocal laser scanning microscope with excitation at $405 \mathrm{~nm}$ using a diode laser, and GFP fluorescence recorded between 506 and $589 \mathrm{~nm}$. The images shown were taken using a $63 \times$ objective with 1.64 zoom, in $0.2 \mu \mathrm{m}$ steps and set at $1024 \times 1024$ pixels.

\section{Conclusions}

Whether DotC has a role in dothistromin efflux from the cell remains inconclusive. Firstly although mutants deficient in DotC secreted less dothistromin, and a slightly lower \% of total dothistromin, than wild types, these results could have been due to overall lower levels of dothistromin biosynthesis. Secondly although DotC-GFP fusions suggested that DotC is located on the cell membrane, DotC does not appear to be the primary efflux transporter as $\operatorname{dotC}$ mutants can still secrete dothistromin. Furthermore a DotC-overproducing strain appeared unable to pump out the very high levels of dothistromin it produced, and had high intracellular toxin levels, suggesting rate limitation by other efflux systems.

In general, transcription of dothistromin genes and dothistromin production decreased in DotC-deficient mutants and increased in a DotC-overproducing strain, suggesting an important role for 
DotC in regulation of dothistromin biosynthesis. Cellular localization studies supported the hypothesis that dothistromin biosynthesis occurs in vacuoles or vesicles as previously suggested for aflatoxin biosynthesis. It is possible that compartmentalization of dothistromin provides D. septosporum with a mechanism of self-protection against its own toxin, and may be the key to understanding how efflux pumps can contribute to the regulation of toxin biosynthesis.

\section{Acknowledgements}

This work was partly supported by the New Zealand National Centre for Advanced Bio-Protection Technologies and was carried out in compliance with the current laws governing genetic experimentation in New Zealand. We thank Dmitri Sokolov (Massey University) for assistance with confocal analysis, Bill Jones (HortResearch, NZ) for providing ELISA reagents and Tim Owen (Massey University) for purification of dothistromin. Y.Y. acknowledges funding from the China Scholarship Council under the visiting scholar program.

\section{References}

1. Bassett, C.; Buchanan, M.; Gallagher, R.T.; Hodges, R.L. A toxic difuroanthraquinone from Dothistroma pini. Chem. Ind. 1970, 52, 1659-1660.

2. Bradshaw, R.E. Dothistroma (red-band) needle blight of pines and the dothistromin toxin: A review. Forest Pathol. 2004, 34, 163-185.

3. Barnes, I.; Crous, P.W.; Wingfield, B.D.; Wingfield, M.J. Multigene phylogenies reveal that red band needle bight of Pinus is caused by two distinct species of Dothistroma, D. septosporum and D. pini. Stud. Mycol. 2004, 50, 551-565.

4. Woods, A.J.; Coates, K.D.; Hamann, A. Is an unprecedented Dothistroma needle blight epidemic related to climate change? Bioscience 2005, 55, 761-769.

5. Brown, A.; Webber, J.F. Red Band Needle Blight in Conifers in Britain; Forestry Commission: Farnham, Surrey, UK, June 2008; pp. 1-8.

6. Bradshaw, R.E.; Zhang, S. Biosynthesis of dothistromin. Mycopathologia 2006, 162, 201-213.

7. Stoessl, A.; Abramowski, Z.; Lester, H.H.; Rock, G.L.; Towers, G.H.N. Further toxic properties of the fungal metabolite dothistromin. Mycopathologia 1990, 112, 179-186.

8. Jones, W.T.; Harvey, D.; Jones, S.D.; Sutherland, P.W.; Nicol, M.J.; Sergejew, N.; Debnam, P.M.; Cranshaw, N.; Reynolds, P.H.S. Interaction between the phytotoxin dothistromin and Pinus radiata embryos. Phytopathology 1995, 85, 1099-1104.

9. Youngman, R.J.; Elstner, E.F. Photodynamic and reductive mechanisms of oxygen activation by the fungal phytotoxins, cercosporin and dothistromin. In Oxygen Radicals in Chemistry and Biology; Bors, W., Saran, M., Eds.; Walter de Gruyter and Co.: Berlin, Germany, 1984; pp. 501-508.

10. Schwelm, A.; Barron, N.J.; Baker, J.; Dick, M.; Long, P.G.; Zhang, S.; Bradshaw, R.E. Dothistromin toxin is not required for dothistroma needle blight in Pinus radiata. Plant Pathol. 2009, 58, 293-304.

11. Del Sorbo, G.; Schoonbeek, H.; de Waard, M.A. Fungal transporters involved in efflux of natural toxic compound and fungicides. Fungal Genet. Biol. 2000, 30, 1-15. 
12. Coleman, J.J.; Mylonakis, E. Efflux in Fungi: La Pièce de Résistance. PLoS Pathogens 2009, 5, e1000486.

13. Callahan, T.M.; Rose, M.S.; Meade, M.J.; Ehrenshaft, M.; Upchurch, R.G. CFP, the putative cercosporin transporter of Cercospora kikuchii, is required for wild type cercosporin production, resistance and virulence on soybean. Mol. Plant Microbe Interact. 1999, 12, 901-910.

14. Choquer, M.; Lee, M.H.; Bau, H.J.; Chung, K.R. Deletion of a MFS transporter-like gene in Cercospora nicotianae reduces cercosporin toxin accumulation and fungal virulence. FEBS Lett. 2007, 581, 489-494.

15. Upchurch, R.G.; Rose, M.S.; Eweida, M. Over-expression of the cercosporin facilitator protein, CFP, in Cercospora kikuchii up-regulates production and secretion of cercosporin. FEMS Microbiol. Lett. 2001, 204, 89-93.

16. Chang, P.-K.; Yu, J.; Yu, J.-H. aflT, a MFS transporter-encoding gene located in the aflatoxin gene cluster, does not have a significant role in aflatoxin secretion. Fungal Genet. Biol. 2004, 41, 911-920.

17. Amnuaykanjanasin, A.; Daub, M.E. The ABC transporter ATR1 is necessary for efflux of the toxin cercosporin in the fungus Cercospora nicotianae. Fungal Genet. Biol. 2009, 46, 146-158.

18. Bradshaw, R.E.; Bhatnagar, D.; Ganley, R.J.; Gillman, C.J.; Monahan, B.J.; Seconi, J.M. Dothistroma pini, a forest pathogen, contains homologs of aflatoxin biosynthetic pathway genes. Appl. Environ. Microbiol. 2002, 68, 2885-2892.

19. Zhang, S.; Schwelm, A.; Jin, H.P.; Collins, L.J.; Bradshaw, R.E. A fragmented aflatoxin-like gene cluster in the forest pathogen Dothistroma septosporum. Fungal Genet. Biol. 2007, 44, 13421354.

20. Bradshaw, R.E.; Jin, H.P.; Morgan, B.S.; Schwelm, A.; Teddy, O.R.; Young, C.A.; Zhang, S.G. A polyketide synthase gene required for biosynthesis of the aflatoxin-like toxin, dothistromin. Mycopathologia 2006, 161, 283-294.

21. Hong, S.Y.; Linz, J.E. Functional expression and subcellular localization of the aflatoxin pathway enzyme Ver-1 fused to enhanced green fluorescent protein. Appl. Environ. Microbiol. 2008, 74, 6385-6396.

22. Hong, S.-Y.; Linz, J.E. Functional expression and sub-cellular localization of the early aflatoxin pathway enzyme Nor-1 in Aspergillus parasiticus. Mycol. Res. 2009, 113, 591-601.

23. Chanda, A.; Roze, L.V.; Pastor, A.; Frame, M.K.; Linz, J.E. Purification of a vesicle-vacuole fraction functionally linked to aflatoxin synthesis in Aspergillus parasiticus. J. Microbiol. Meth. 2009, 78, 28-33.

24. Bradshaw, R.E.; Ganley, R.J.; Jones, W.T.; Dyer, P.S. High levels of dothistromin toxin produced by the forest pathogen Dothistroma pini. Mycol. Res. 2000, 104, 325-332.

25. Bradshaw, R.E.; Bidlake, A.; Forester, N.; Scott, D.B. Transformation of the fungal forest pathogen Dothistroma pini to hygromycin resistance. Mycol. Res. 1997, 101, 1247-1250.

26. Silar, P. Two new easy to use vectors for transformations. Fungal Genet. Newsl. 1995, 42, 73.

27. Jones, W.T.; Harvey D.; Jones S.D.; Fielder, S.; Debnam, P.; Reynolds, P.H.S. Competitive ELISA employing monoclonal antibodies specific for dothistromin. Food Agric. Immunol. 1993, 5, 187-197. 
28. Schwelm, A.; Barron, N.J.; Zhang, S.; Bradshaw, R.E. Early expression of aflatoxin-like dothistromin genes in the forest pathogen Dothistroma septosporum. Mycol. Res. 2008, 112, $138-146$.

29. Tanaka, A.; Christensen, M.J.; Takemoto, D.; Park, P.; Scott, B. Reactive oxygen species play a role in regulating a fungus-perennial ryegrass mutualistic interaction. Plant Cell 2006, 18, $1052-1066$.

(C) 2009 by the authors; licensee Molecular Diversity Preservation International, Basel, Switzerland. This article is an open-access article distributed under the terms and conditions of the Creative Commons Attribution license (http://creativecommons.org/licenses/by/3.0/). 